\title{
Activity of Cefepime-Zidebactam against Multidrug-Resistant (MDR) Gram-Negative Pathogens
}

\author{
Kenneth S. Thomson ${ }^{1, *} \mathbb{0}$, Sameh AbdelGhani ${ }^{1,2}$, James W. Snyder ${ }^{1}$ and Gina K. Thomson ${ }^{1,3}$ \\ 1 Department of Pathology and Laboratory Medicine, School of Medicine, University of Louisville, \\ Louisville, KY 40202, USA; sameh.abdelghani@louisville.edu (S.A.); james.snyder@louisville.edu (J.W.S.); \\ ginamu@ulh.org (G.K.T.) \\ 2 Faculty of Pharmacy, Beni-Suef University, Beni-Suef 62511, Egypt \\ 3 Microbiology Department, University of Louisville Hospital, Louisville, KY 40202, USA \\ * Correspondence: kenneth.thomson@louisville.edu
}

Received: 18 February 2019; Accepted: 19 March 2019; Published: 23 March 2019

check for updates

\begin{abstract}
This study compared the activity of cefepime + zidebactam (FEP-ZID) and selected currently available antibacterial agents against a panel of multidrug-resistant (MDR) clinical isolates chosen to provide an extreme challenge for antibacterial activity. FEP-ZID had a very broad and potent in vitro spectrum of activity, and was highly active against many MDR isolates of Enterobacterales, Pseudomonas aeruginosa, and Acinetobacter baumannii. Notably, it inhibited isolates producing carbapenemases of Ambler classes A, B, and D, and P. aeruginosa isolates with multiple resistance mechanisms including combinations of upregulated efflux, diminished or non-functional OprD porins, and AmpC overproduction. Its clinical role will be determined initially by the breakpoints assigned to it, comparison studies with other investigational $\beta$-lactamase inhibitor combinations, and ultimately by the developing body of therapeutic outcome data.
\end{abstract}

Keywords: carbapenemase-producing organism; carbapenemase; zidebactam; therapy

\section{Introduction}

Gram-negative multidrug-resistant (MDR) pathogens, particularly carbapenemase-producing organisms (CPOs), cause infections of high mortality that are typically treated with antibiotic combinations that include toxic drugs such as polymyxins and aminoglycosides [1]. Recent Food and Drug Administration (FDA) approvals of the $\beta$-lactamase inhibitor combinations ceftazidime + avibactam and meropenem + vaborbactam have created the potential for safer treatments of infections by CPOs producing Ambler class A carbapenemases, and also pathogens that produce AmpC and/or extended spectrum $\beta$-lactamases [2-6]. Other $\beta$-lactamase inhibitor combinations are under development, e.g., imipenem + relebactam, aztreonam + avibactam, meropenem + nacubactam, cefepime + zidebactam, and cefepime + VNRX-5133, some of which are active against CPOs producing Ambler class A, B, and D carbapenemases [2,4,5,7-13]. The clinical potential of the new $\beta$-lactamase inhibitor combinations is significant, but the optimism about them should be tempered by reports of reduced susceptibility or resistance to ceftazidime + avibactam in clinical isolates sometimes emerging during therapy [14-19]. Arising concerns include whether the ceftazidime + avibactam resistance can impact the effectiveness of the developmental combinations, and whether or not ceftazidime + avibactam resistance indicates the need to avoid suboptimal combinations of co-drugs and inhibitors. For example, ceftazidime was previously associated with the development of resistance when used for treating infections by extended spectrum $\beta$-lactamase (ESBL) nd AmpC producers [20-22]. Therefore, given the benefit of hindsight, it was a questionable choice of co-drug for avibactam. Furthermore, it is possible that avibactam, a diazobicyclooctane, has attributes that may prove to be suboptimal in 
that various types of $\beta$-lactamases may develop resistance to it $[19,23,24]$, and its clinical spectrum of carbapenemase coverage is limited to class A and possibly OXA-48-like class D carbapenemases [7]. Class B carbapenemases and the class D carbapenemases of Acinetobacter spp. are gaps in its spectrum. It is also currently unknown if resistance to avibactam may confer resistance to its diazobicyclooctane analogues, relebactam and nacubactam.

Cefepime + zidebactam (FEP-ZID) is a unique combination agent in that its bicyclo-acyl hydrazide component, zidebactam, is a $\beta$-lactamase inhibitor which possesses intrinsic antibacterial activity. That is, zidebactam can both protect cefepime from hydrolysis by $\beta$-lactamases and extend its spectrum of antibacterial activity $[12,13]$. With the aim of assessing its activity against Gram-negative isolates chosen to provide an extreme challenge of antibacterial activity, a study was designed to evaluate the in vitro activity of FEP-ZID and selected currently available antibacterial agents against a panel of well-characterized MDR clinical isolates, with a special focus on diverse types of carbapenemase producers.

\section{Methodology}

\subsection{Isolates}

The study isolates consisted of 132 isolates, comprising of Enterobacterales ( $n=102)$, Pseudomonas aeruginosa $(n=18)$, and Acinetobacter baumannii $(n=12)$ that were previously characterized by molecular, phenotypic, and biochemical tests for types of $\beta$-lactamase production. Some P. aeruginosa isolates were also characterized for degree of efflux and porin expression. These isolates were donated by the Centers for Disease Control and Prevention, Food and Drug Administration Antimicrobial Resistance Isolate Bank, and the Microbiology Laboratory at the University of Louisville Hospital. The study also includes isolates from previously published studies $[25,26]$. The specific resistance mechanisms for the three groups of organisms (Enterobacterales, P. aeruginosa, and A. baumannii) are provided in Table 1 and Supplementary Tables $\mathrm{S} 1$ and $\mathrm{S} 2$ for individual isolates. The class A carbapenemases, produced by 39 isolates, included KPC-2, KPC-3, KPC-4, KPC-5, KPC-6, KPC-8, KPC-18, NMC-A, SME-type, and GES-5. The class B carbapenemases, produced by 28 isolates, included IMP-1, IMP-7, IMP-8, VIM-1, VIM-2, VIM-4, VIM-7, NDM-1, GIM-1, and SPM-1, and the class D carbapenemases, produced by 22 isolates, included OXA-23, OXA-40, OXA-58, OXA-48, OXA-181, and OXA-232. Five CPOs produced two classes of carbapenemases. ESBLs that were produced by 22 non-CPO isolates of Enterobacteriaceae included CTX-M-1, CTX-M-2, CTX-M-9, CTX-M-12, CTX-M-14, CTX-M-15, CTX-M-18, CTX-M-28, CTX-M-45, TEM-16, SHV-3, SHV-4, SHV-7, and SHV-12, with some isolates co-producing an AmpC $\beta$-lactamase. Ten isolates of Enterobacterales produced plasmid-mediated AmpCs of CMY, DHA, FOX, and LAT types. The above-mentioned $\beta$-lactamases are ones capable of causing resistance to at least one of the study drugs. Types of isolates included in the study but not included in the above groups either produced limited-spectrum $\beta$-lactamases or had other resistance mechanisms, such as reduced permeability or upregulated efflux. 
Table 1. Resistance mechanisms of organism groups.

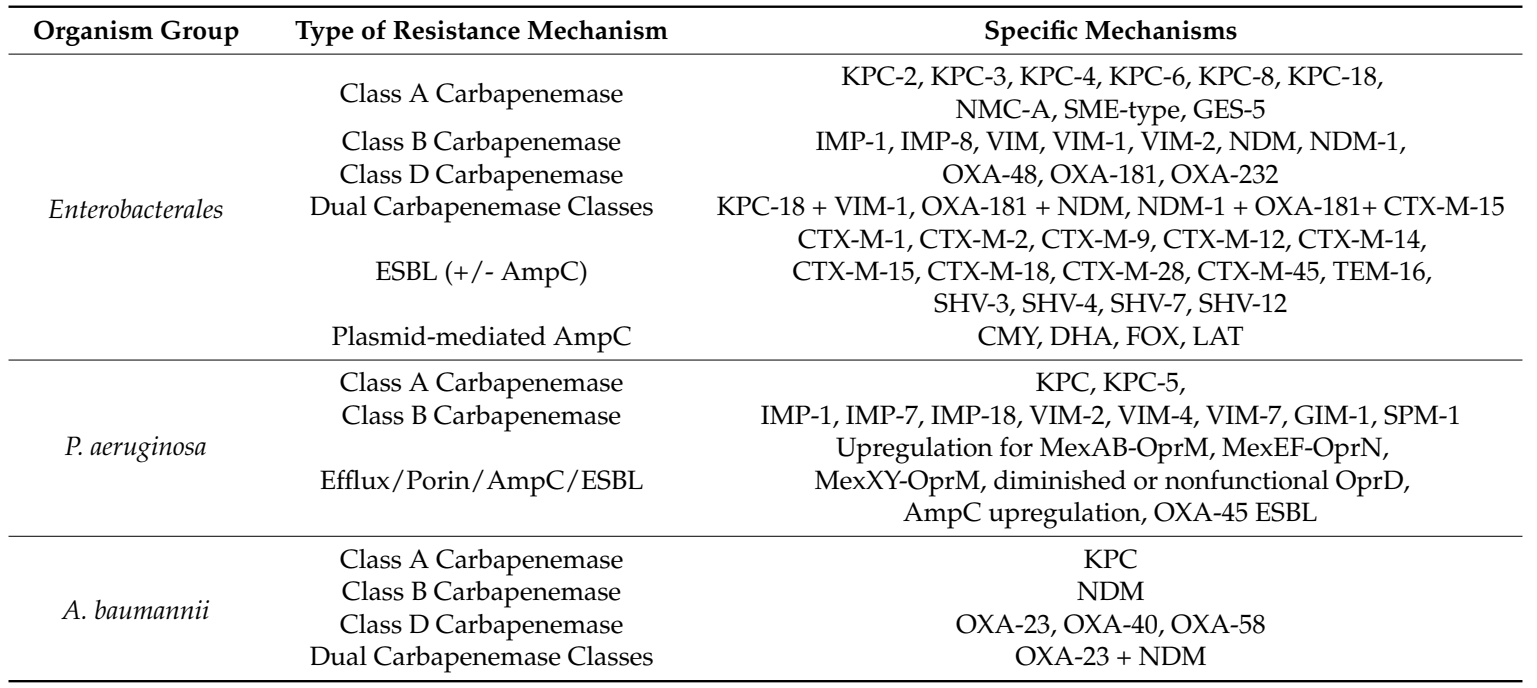

\subsection{Susceptibility Testing}

Antibiotic susceptibility test results were reported as minimum inhibitory concentrations (MICs), and interpreted against breakpoints where possible. Breakpoints are specific concentrations of antibacterial agents considered to be predictive of therapeutic responses to normally attained tissue of body fluid concentrations. They are typically described as susceptible (agent is likely to be clinically active), resistant (agent is likely to be ineffective), and intermediate (activity uncertain when agent is dosed as recommended, but may be effective at higher doses). The in vitro activities of FEP-ZID, piperacillin + tazobactam, ceftolozane + tazobactam, cefepime, imipenem, ceftazidime, levofloxacin, amikacin, and polymyxin B were determined by Clinical and Laboratory Standards Institute (CLSI) agar dilution methodology, using doubling dilutions of antibacterial agents incorporated in Mueller-Hinton agar (BD Diagnostics, Sparks, MD, USA), inocula of $10^{4}$ colony forming units (cfu)/spot, and overnight incubation at $35^{\circ} \mathrm{C}$. Concentrations of zidebactam and $\beta$-lactamase inhibitors comprised a fixed $4 \mu \mathrm{g} / \mathrm{mL}$ of tazobactam for ceftolozane + tazobactam and piperacillin + tazobactam, and a 1:1 ratio combination for FEP-ZID and cefoperazone + sulbactam. MICs of $\beta$-lactamase inhibitor combinations were reported for only the $\beta$-lactam co-drug. Where applicable, susceptibility was interpreted using appropriate CLSI breakpoints for relevant organism groups [27]. The CLSI high-dose susceptible breakpoint of $\leq 8 \mu \mathrm{g} / \mathrm{mL}$ was used for cefepime when tested alone. In the absence of CLSI breakpoints, susceptibility was not interpreted for tests with FEP-ZID and cefoperazone + sulbactam. The control strains were Escherichia coli ATCC 25922, E. coli ATCC 35218, Klebsiella pneumoniae ATCC 700603, and P. aeruginosa ATCC 27853. Results were summarized as the concentrations that inhibited $50 \%$ and $90 \%$ of isolates $\left(\mathrm{MIC}_{50}\right.$ and $\mathrm{MIC}_{90}$ values, respectively). Percent susceptibility was calculated only for organism groups with a minimum of 10 isolates.

The sources of the laboratory powders of antibacterial agents were: Wockhardt Research Center Aurangabad, India (cefepime, ceftolozane, cefoperazone, zidebactam, and tazobactam); Thermo Fisher Scientific Chemicals Inc., Ward Hill, MA, USA (imipenem and amikacin); TCI America, Portland, OR, USA (levofloxacin, piperacillin, and ceftazidime); and VWR International, LLC, Batavia, IL, USA (polymyxin B).

\section{Results}

\subsection{Activity against All Isolates}

As shown in Table 2, FEP-ZID and polymyxin B were the most potent agents against this collection of predominantly carbapenemase-producing isolates. The $\mathrm{MIC}_{50}$ and $\mathrm{MIC}_{90}$ values for 
FEP-ZID were 0.5 and $16 \mu \mathrm{g} / \mathrm{mL}$, respectively, and they were 1 and $\geq 16 \mu \mathrm{g} / \mathrm{mL}$, respectively, for polymyxin B. With the exception of imipenem ( $\mathrm{MIC}_{50}$ and $\mathrm{MIC}_{90}$ values 4 and $128 \mu \mathrm{g} / \mathrm{mL}$, respectively), all other comparison agents had 32 to 256-fold higher $\mathrm{MIC}_{50}$ values than FEP-ZID and high off-scale $\mathrm{MIC}_{90}$ values.

Table 2. Potencies against all isolates.

\begin{tabular}{|c|c|c|c|}
\hline \multirow{2}{*}{ Agent } & \multicolumn{3}{|c|}{ Results Against All 132 Isolates in $\mu \mathrm{g} / \mathrm{mL}$} \\
\hline & MIC Range & MIC50 & MIC90 \\
\hline FEP-ZID & $0.03-\geq 128$ & 0.5 & 16 \\
\hline Cefepime & $0.03-\geq 128$ & 16 & $\geq 128$ \\
\hline Cefoperazone + sulbactam & $\leq 0.25-\geq 64$ & 16 & $\geq 64$ \\
\hline Ceftolozane + tazobactam & $\leq 0.06-\geq 64$ & 32 & $\geq 64$ \\
\hline Piperacillin + tazobactam & $0.5-\geq 256$ & 128 & $\geq 256$ \\
\hline Ceftazidime & $\leq 0.06-\geq 256$ & 128 & $\geq 256$ \\
\hline Imipenem & $\leq 0.06-\geq 256$ & 4 & $\overline{128}$ \\
\hline Amikacin & $\leq 0.25-\geq 256$ & 16 & $\geq 256$ \\
\hline Levofloxacin & $0.03-\geq 32$ & 16 & $\geq 32$ \\
\hline Polymyxin B & $0.25-\geq 16$ & 1 & $\geq 16$ \\
\hline
\end{tabular}

\subsection{Activity against CPOs}

In tests with all CPOs, FEP-ZID was the most potent agent, with respective $\mathrm{MIC}_{50}$ and $\mathrm{MIC}_{90}$ values of 1 and $16 \mu \mathrm{g} / \mathrm{mL}$. Polymyxin B was the next most potent agent, with $\mathrm{MIC}_{50}$ and $\mathrm{MIC}_{90}$ values of 1 and $\geq 16 \mu \mathrm{g} / \mathrm{mL}$ (Table 2). Against isolates producing class A carbapenemases (Table S1), FEP-ZID and polymyxin $\mathrm{B}$ had respective $\mathrm{MIC}_{50}$ and $\mathrm{MIC}_{90}$ values of 0.5 and $8 \mu \mathrm{g} / \mathrm{mL}$, and 1 and $\geq 16 \mu \mathrm{g} / \mathrm{mL}$. Cefepime ( $\mathrm{MIC}_{50}$ and $\mathrm{MIC}_{90}$ values of 4 and $\geq 128 \mu \mathrm{g} / \mathrm{mL}$, respectively) and amikacin $\left(\mathrm{MIC}_{50}\right.$ and $\mathrm{MIC}_{90}$ values of 8 and $64 \mu \mathrm{g} / \mathrm{mL}$, respectively) were the only other agents exhibiting activity against more than $50 \%$ of the class A CPOs (both $56.1 \%$ susceptibility). Isolates producing class B carbapenemases were more resistant, but FEP-ZID $\left(\mathrm{MIC}_{50}\right.$ and $\mathrm{MIC}_{90}$ values of 2 and $32 \mu \mathrm{g} / \mathrm{mL}$, respectively) was distinctly more active than the comparison agents, all of which had high, off-scale $\mathrm{MIC}_{90}$ values, with very few isolates being susceptible. Similarly, against isolates producing class D carbapenemases, with the exception of polymyxin B, FEP-ZID, with MIC $_{50}$ and MIC 90 values of 2 and $16 \mu \mathrm{g} / \mathrm{mL}$, respectively, was distinctly more active than the comparison agents. With the exception of one isolate of OXA-48-producing K. pneumoniae (MIC $\geq 128 \mu \mathrm{g} / \mathrm{mL}$ ), all other Enterobacterales producing OXA-48-like carbapenemases had FEP-ZID MICs of $\leq 2 \mu \mathrm{g} / \mathrm{mL}$. OXA-carbapenemase-producing isolates of A. baumannii were more resistant overall than OXA-producing Enterobacterales. A. baumannii isolates producing OXA-23 or OXA-40 had FEP-ZID MICs in the range 4 to $32 \mu \mathrm{g} / \mathrm{mL}$. One isolate producing two carbapenemases (OXA-23 + NDM) had a FEP-ZID MIC of $\geq 128 \mu \mathrm{g} / \mathrm{mL}$. All other dual carbapenemase producers had FEP-ZID MICs of $\leq 2 \mu \mathrm{g} / \mathrm{mL}$.

\subsection{Activity against ESBL-Producing and Plasmid-Mediated AmpC-Producing Enterobacterales}

FEP-ZID, polymyxin B, and imipenem were the most active agents against ESBL-producing Enterobacterales. Only two agents, FEP-ZID and polymyxin B, had low $\mathrm{MIC}_{90}$ values $(2 \mu \mathrm{g} / \mathrm{mL}$ for both agents). Imipenem was the next most active agent (MIC 90 value of $16 \mu \mathrm{g} / \mathrm{mL}$ and $83.4 \%$ susceptibility). All other comparison agents had high, off-scale $\mathrm{MIC}_{90}$ values. Among these, only amikacin (72.7\%), ceftolozane + tazobactam (59.1\%), and piperacillin + tazobactam (59.1\%) inhibited more than 50\% of the ESBL producers.

Against the plasmid-mediated AmpC-producing Enterobacterales, FEP-ZID ( $\mathrm{MIC}_{50}$ and $\mathrm{MIC}_{90}$ values of 0.12 and $2 \mu \mathrm{g} / \mathrm{mL}$, respectively) and cefepime ( $\mathrm{MIC}_{50}$ and $\mathrm{MIC}_{90}$ values of $0.5 \mathrm{and} 8 \mu \mathrm{g} / \mathrm{mL}$, respectively, and $90 \%$ susceptibility) were the most active agents. Polymyxin B was the next most active agent $\left(\mathrm{MIC}_{50}\right.$ and $\mathrm{MIC}_{90}$ values were both $\left.1 \mu \mathrm{g} / \mathrm{mL}\right)$. Eight of the 10 plasmid-mediated 
AmpC-producing isolates had FEP-ZID MICs of $\leq 0.25 \mu \mathrm{g} / \mathrm{mL}$. The agents most compromised by plasmid-mediated AmpCs were ceftolozane + tazobactam, piperacillin + tazobactam, and levofloxacin.

\subsection{Activity against MDR Enterobacterales, P. aeruginosa, and A. baumannii Irrespective of Resistance Mechanisms}

FEP-ZID was the most active agent against all Enterobacterales, with a dramatically lower $\mathrm{MIC}_{90}$ value $(2 \mu \mathrm{g} / \mathrm{mL})$ than the other study drugs ( $128 \mu \mathrm{g} / \mathrm{mL}$ or high, off-scale values). Of the drugs for which breakpoints were available, the most active were amikacin ( $58.8 \%$ susceptible) and imipenem (47.1\% susceptible).

Against all $P$. aeruginosa isolates, polymyxin B was the most potent agent, with $\mathrm{MIC}_{50}$ and $\mathrm{MIC}_{90}$ values of 2 and $4 \mu \mathrm{g} / \mathrm{mL}$, respectively, followed by FEP-ZID ( $\mathrm{MIC}_{50}$ and $\mathrm{MIC}_{90}$ values of 8 and $32 \mu \mathrm{g} / \mathrm{mL}$, respectively). With the exceptions of amikacin and polymyxin B (both 61.1\% susceptibility), the other agents inhibited less than $40 \%$ of the isolates at the CLSI susceptible breakpoints. Agents with most potency against the more susceptible P. aeruginosa isolates, indicated by $\mathrm{MIC}_{50}$ values, were polymyxin B $(2 \mu \mathrm{g} / \mathrm{mL})$, FEP-ZID $(8 \mu \mathrm{g} / \mathrm{mL})$, ceftolozane + tazobactam $(16 \mu \mathrm{g} / \mathrm{mL})$, and amikacin $(16 \mu \mathrm{g} / \mathrm{mL})$. Reduced susceptibility to FEP-ZID was either associated with IMP or VIM class $\mathrm{B}$ carbapenemases, or combinations of mechanisms such as overexpressed MexAB-OprM or MexXY efflux, diminished OprD function, and high-level AmpC production.

Polymyxin B ( $\mathrm{MIC}_{50}$ and $\mathrm{MIC}_{90}$ values of 1 and $2 \mu \mathrm{g} / \mathrm{mL}$, respectively, and $91.2 \%$ susceptibility) and FEP-ZID ( $\mathrm{MIC}_{50}$ and $\mathrm{MIC}_{90}$ values of 16 and $32 \mu \mathrm{g} / \mathrm{mL}$, respectively) were the most active agents against $A$. baumannii. On the basis of CLSI breakpoints, amikacin ( $50.0 \%$ susceptibility) was the next most active agent, with the other drugs with breakpoints having susceptibility rates of only $0 \%$ to $8.3 \%$.

\section{Discussion}

This study comprised a very challenging panel of isolates. The diversity of the range of single-carbapenemase and dual-carbapenemase production provided the strongest challenge for FEP-ZID activity in this study, and thereby extended our understanding of the activity of FEP-ZID and provided insights into its potential against MDR isolates of Enterobacterales, P. aeruginosa, and A. baumannii. FEP-ZID was notable for its activity against CPOs producing all three Ambler classes of carbapenemases and against $P$. aeruginos $a$ with multiple resistance mechanisms, including combinations of upregulated efflux, diminished or non-functional OprD porins, and AmpC overproduction. Especially notable was the ability of zidebactam to enhance the activity of cefepime against CPOs producing class $\mathrm{D}$ carbapenemases. Nontoxic treatment for infections by MDR A. baumannii isolates producing OXA class $\mathrm{D}$ carbapenemases is currently an unmet medical need. There is also a need for effective therapies for infections by MDR isolates of $P$. aeruginosa [28,29]. The activity of FEP-ZID against such isolates warrants clinical investigation.

If the potency of FEP-ZID translates into clinical efficacy in humans, FEP-ZID could be considered as an expanded spectrum $\beta$-lactam/ $\beta$-lactam enhancer combination. This is consistent with previous reports $[12,13,30]$. The need for FEP-ZID breakpoints and recommended testing methods is urgent, because the CPO problem is global and associated with high mortality [31-35]. There are no drugs to treat all $\mathrm{CPO}$ infections, and resistance continues to emerge. It is essential to reduce the mortality and control the spread of these organisms, and it must be done now. The need for effective therapies is urgent. To avoid delays from hindering the quest for effective therapy of all types of CPO infections, recent surveillance data, population pharmacokinetic (PK), Monte Carlo-based pharmacokinetic/pharmacodynamic (PK/PD) studies, and animal models utilizing human-simulated dosing regimens against high MIC strains can be analyzed to expedite breakpoint and testing recommendations.

At this time, in the absence of FEP-ZID breakpoints, we avoided making interpretations of susceptibility or resistance in our analysis, and solely reported in vitro potency data for this agent. 


\section{Conclusions}

In conclusion, FEP-ZID exhibited broad spectrum potency against a wide range of MDR Gram-negative pathogens in this study. Depending on the breakpoints that are assigned, its antibacterial spectrum may provide more comprehensive CPO coverage for empirical therapy of infections than the current limited-spectrum agents, ceftazidime + avibactam and meropenem + vaborbactam. The clinical role of FEP-ZID will be determined initially by the breakpoints assigned to it, followed by comparison studies with other investigational $\beta$-lactamase inhibitor combinations and, ultimately, by the developing body of therapeutic outcome data.

Supplementary Materials: The following are available online at http:/ / www.mdpi.com/2079-6382/8/1/32/s1, Table S1: Activity of FEP-ZID and Comparison Agents, Table S2: MIC of FEP-ZID and Comparators against All Tested Strains.

Author Contributions: Conceptualization, K.S.T. and G.K.T.; methodology, K.S.T. and G.K.T.; software, S.A.; formal analysis, K.S.T. and G.K.T.; investigation, K.S.T., G.K.T. and S.A.; writing一original draft preparation, K.S.T. and G.K.T.; writing-review and editing, K.S.T., G.K.T., J.W.S. and S.A.; supervision, K.S.T.; project administration, K.S.T., G.K.T., and J.W.S.; funding acquisition, K.S.T.

Acknowledgments: We thank Wockhardt Bio AG, Switzerland, for funding this study and providing some of the laboratory powders of antibacterial agents.

Conflicts of Interest: The authors declare no conflict of interest.

\section{References}

1. Tzouvelekis, L.S.; Markogiannakis, A.; Piperaki, E.; Souli, M.; Daikos, G.L. Treating infections caused by carbapenemase-producing Enterobacteriaceae. Clin. Microbiol. Infect. 2014, 20, 862-872. [CrossRef] [PubMed]

2. Dupont, H.; Gaillot, O.; Goetgheluck, A.S.; Plassart, C.; Emond, J.P.; Lecuru, M.; Gaillard, N.; Derdouri, S.; Lemaire, B.; Girard de Courtilles, M.; et al. Molecular Characterization of Carbapenem-Nonsusceptible Enterobacterial Isolates Collected during a Prospective Interregional Survey in France and Susceptibility to the Novel Ceftazidime-Avibactam and Aztreonam-Avibactam Combinations. Antimicrob. Agents Chemother. 2016, 60, 215-221. [CrossRef] [PubMed]

3. Lapuebla, A.; Abdallah, M.; Olafisoye, O.; Cortes, C.; Urban, C.; Quale, J.; Landman, D. Activity of Meropenem Combined with RPX7009, a Novel beta-Lactamase Inhibitor, against Gram-Negative Clinical Isolates in New York City. Antimicrob. Agents Chemother. 2015, 59, 4856-4860. [CrossRef] [PubMed]

4. Beidenbach, D.J.; Kazmierczak, K.; Bouchillon, S.K.; Sahm, D.; Bradford, P.A. In Vitro Activity of Aztreonam-Avibactam against a Global Collection of Gram-Negative Pathogens from 2012 and 2013. Antimicrob. Agents Chemother. 2015, 59, 4239-4248. [CrossRef] [PubMed]

5. Li, H.; Estabrook, M.; Jacoby, G.A.; Nichols, W.W.; Testa, R.T.; Bush, K. In Vitro Susceptibility of Characterized Beta-Lactamase-Producing Strains Tested with Avibactam Combinations. Antimicrob. Agents Chemother. 2015, 59, 1789-1793. [CrossRef]

6. Castanheira, M.; Huband, M.D.; Mendes, R.E.; Flamm, R.K. Meropenem-Vaborbactam Tested against Contemporary Gram-Negative Isolates Collected Worldwide during 2014, Including Carbapenem-Resistant, KPC-Producing, Multidrug-Resistant, and Extensively Drug-Resistant Enterobacteriaceae. Antimicrob. Agents Chemother. 2017, 61. [CrossRef] [PubMed]

7. Vasoo, S.; Cunningham, S.A.; Kohner, P.C.; Simner, P.J.; Mandrekar, J.N.; Lolans, K.; Hayden, M.K.; Patel, R. Comparison of a novel, rapid chromogenic biochemical assay, the Carba NP test, with the modified Hodge test for detection of carbapenemase-producing Gram-negative bacilli. J. Clin. Microbiol. 2013, 51, 3097-4101. [CrossRef]

8. Singh, R.; Kim, A.; Tanudra, M.A.; Harris, J.J.; McLaughlin, R.E.; Patey, S.; O’Donnell, J.P.; Bradford, P.A.; Eakin, A.E. Pharmacokinetics/pharmacodynamics of a beta-lactam and beta-lactamase inhibitor combination: A novel approach for aztreonam/avibactam. J. Antimicrob. Chemother. 2015, 70, 2618-2626. [CrossRef]

9. Lapuebla, A.; Abdallah, M.; Olafisoye, O.; Cortes, C.; Urban, C.; Landman, D.; Quale, J. Activity of Imipenem with Relebactam against Gram-Negative Pathogens from New York City. Antimicrob. Agents Chemother. 2015, 59, 5029-5031. [CrossRef] 
10. Hirsch, E.B.; Ledesma, K.R.; Chang, K.T.; Schwartz, M.S.; Motyl, M.R.; Tam, V.H. In vitro activity of MK-7655, a novel beta-lactamase inhibitor, in combination with imipenem against carbapenem-resistant Gram-negative bacteria. Antimicrob. Agents Chemother. 2012, 56, 3753-3757. [CrossRef]

11. Georgiou, P.-C.; Siopi, M.; Tsala, M.; Lagarde, C.; Kloezen, W.; Donnelly, R.; Meletiadis, J.; Mouton, J. VNRX-5133, a novel broad-spectrum beta-lactamase inhibitor, enhances the activity of cefepime against resistant Enterobacteriaceae and P. aeruginosa isolates in a neutropenic mouse-thigh infection model, abstr. In Proceedings of the 28th European Congress of Clinical Microbiology and Infectious Diseases, Madrid, Spain, 21-24 April, 2018.

12. Moya, B.; Barcelo, I.M.; Bhagwat, S.; Patel, M.; Bou, G.; Papp-Wallace, K.M.; Bonomo, R.A.; Oliver, A. Potent Beta-Lactam Enhancer Activity of Zidebactam and WCK 5153 against Acinetobacter baumannii, Including Carbapenemase-Producing Clinical Isolates. Antimicrob. Agents Chemother. 2017, 61, e01238-17. [CrossRef] [PubMed]

13. Moya, B.; Barcelo, I.M.; Bhagwat, S.; Patel, M.; Bou, G.; Papp-Wallace, K.M.; Bonomo, R.A.; Oliver, A. WCK 5107 (Zidebactam) and WCK 5153 Are Novel Inhibitors of PBP2 Showing Potent "Beta-Lactam Enhancer" Activity against Pseudomonas aeruginosa, Including Multidrug-Resistant Metallo-Beta-Lactamase-Producing High-Risk Clones. Antimicrob. Agents Chemother. 2017, 61, e02529-16. [CrossRef] [PubMed]

14. Shields, R.K.; Clancy, C.J.; Hao, B.; Chen, L.; Press, E.G.; Iovine, N.M.; Kreiswirth, B.N.; Nguyen, M.H. Effects of Klebsiella pneumoniae carbapenemase subtypes, extended-spectrum beta-lactamases, and porin mutations on the in vitro activity of ceftazidime-avibactam against carbapenem-resistant K. pneumoniae. Antimicrob. Agents Chemother. 2015, 59, 5793-5797. [CrossRef] [PubMed]

15. Shields, R.K.; Potoski, B.A.; Haidar, G.; Hao, B.; Doi, Y.; Chen, L.; Press, E.G.; Kreiswirth, B.N.; Clancy, C.J.; Nguyen, M.H. Clinical Outcomes, Drug Toxicity, and Emergence of Ceftazidime-Avibactam Resistance Among Patients Treated for Carbapenem-Resistant Enterobacteriaceae Infections. Clin. Infect. Dis. 2016, 63, 1615-1618. [CrossRef] [PubMed]

16. Humphries, R.M.; Hemarajata, P. Resistance to Ceftazidime-Avibactam in Klebsiella pneumoniae Due to Porin Mutations and the Increased Expression of KPC-3. Antimicrob. Agents Chemother. 2017, 61. [CrossRef] [PubMed]

17. Nelson, K.; Hemarajata, P.; Sun, D.; Rubio-Aparicio, D.; Tsivkovski, R.; Yang, S.; Sebra, R.; Kasarskis, A.; Nguyen, H.; Hanson, B.M.; et al. Humphries RM. Resistance to Ceftazidime-Avibactam Is Due to Transposition of KPC in a Porin-Deficient Strain of Klebsiella pneumoniae with Increased Efflux Activity. Antimicrob. Agents Chemother. 2017, 61. [CrossRef] [PubMed]

18. Aitken, S.L.; Tarrand, J.J.; Deshpande, L.M.; Tverdek, F.P.; Jones, A.L.; Shelburne, S.A.; Prince, R.A.; Bhatti, M.M.; Rolston, K.V.; Jones, R.N.; et al. High Rates of Nonsusceptibility to Ceftazidime-avibactam and Identification of New Delhi Metallo-beta-lactamase Production in Enterobacteriaceae Bloodstream Infections at a Major Cancer Center. Clin. Infect. Dis. 2016, 63, 954-958. [CrossRef] [PubMed]

19. Shields, R.K.; Nguyen, M.H.; Press, E.G.; Chen, L.; Kreiswirth, B.N.; Clancy, C.J. Emergence of Ceftazidime-Avibactam Resistance and Restoration of Carbapenem Susceptibility in Klebsiella pneumoniae Carbapenemase-Producing K pneumoniae: A Case Report and Review of Literature. Open Forum Infect. Dis. 2017, 4. [CrossRef] [PubMed]

20. Jacoby, G.A. AmpC beta-lactamases. Clin. Microbiol. Rev. 2009, 22, 161-182. [CrossRef]

21. Livermore, D.M.; Andrews, J.M.; Hawkey, P.M.; Ho, P.L.; Keness, Y.; Doi, Y.; Paterson, D.; Woodford, N. Are susceptibility tests enough, or should laboratories still seek ESBLs and carbapenemases directly? J. Antimicrob. Chemother. 2012, 67, 1569-1577. [CrossRef] [PubMed]

22. Thomson, K.S. Extended-spectrum-beta-lactamase, AmpC, and Carbapenemase issues. J. Clin. Microbiol. 2010, 48, 1019-1025. [CrossRef]

23. Winkler, M.L.; Papp-Wallace, K.M.; Taracila, M.A.; Bonomo, R.A. Avibactam and inhibitor-resistant SHV beta-lactamases. Antimicrob. Agents Chemother. 2015, 59, 3700-3709. [CrossRef] [PubMed]

24. Lahiri, S.D.; Giacobbe, R.A.; Johnstone, M.R.; Alm, R.A. Activity of avibactam against Enterobacter cloacae producing an extended-spectrum class C beta-lactamase enzyme. J. Antimicrob. Chemother. 2014, 69, 2942-2946. [CrossRef]

25. Thomson, G.; Turner, D.; Brasso, W.; Kircher, S.; Guillet, T.; Thomson, K. High-Stringency Evaluation of the Automated BD Phoenix CPO Detect and Rapidec Carba NP Tests for Detection and Classification of Carbapenemases. J. Clin. Microbiol. 2017, 55, 3437-3443. [CrossRef] [PubMed] 
26. Moland, E.S.; Hanson, N.D.; Black, J.A.; Hossain, A.; Song, W.; Thomson, K.S. Prevalence of newer b-lactamases in gram-negative clinical isolates collected in the United States from 2001 to 2002. J. Clin. Microbiol. 2006, 44, 3318-3324. [CrossRef] [PubMed]

27. Clinical and Laboratory Standards Institute. Performance standards for antimicrobial susceptibility testing; twenty-fifth informational supplement M100-ED28; Clinical and Laboratory Standards Institute: Wayne, PA, USA, 2018.

28. Poole, K. Pseudomonas aeruginosa: Resistance to the max. Front. Microbiol. 2011, 2, 65. [CrossRef] [PubMed]

29. Lister, P.D.; Wolter, D.J.; Hanson, N.D. Antibacterial-resistant Pseudomonas aeruginosa: Clinical impact and complex regulation of chromosomally encoded resistance mechanisms. Clin. Microbiol. Rev. 2009, 22, 582-610. [CrossRef] [PubMed]

30. Livermore, D.M.; Mushtaq, S.; Warner, M.; Vickers, A.; Woodford, N. In vitro activity of cefepime/zidebactam (WCK 5222) against Gram-negative bacteria. J. Antimicrob. Chemother. 2017, 72, 1373-1385. [CrossRef]

31. Walsh, T.R. Emerging carbapenemases: A global perspective. Int. J. Antimicrob. Agents 2010, 36, S8-S14. [CrossRef]

32. Walsh, T.R.; Toleman, M.A. The emergence of pan-resistant Gram-negative pathogens merits a rapid global political response. J. Antimicrob. Chemother. 2012, 67, 1-3. [CrossRef]

33. Savard, P.; Perl, T.M. Combating the spread of carbapenemases in Enterobacteriaceae: A battle that infection prevention should not lose. Clin. Microbiol. Infect. 2014, 20, 854-861. [CrossRef] [PubMed]

34. Manenzhe, R.I.; Zar, H.J.; Nicol, M.P.; Kaba, M. The spread of carbapenemase-producing bacteria in Africa: A systematic review. J. Antimicrob. Chemother. 2015, 70, 23-40. [CrossRef] [PubMed]

35. Huttner, A.; Harbarth, S.; Carlet, J.; Cosgrove, S.; Goossens, H.; Holmes, A.; Jarlier, V.; Voss, A.; Pittet, D. Antimicrobial resistance: A global view from the 2013 World Healthcare-Associated Infections Forum. Antimicrob. Resist. Infect. Control 2013, 2, 31. [CrossRef] [PubMed]

(C) 2019 by the authors. Licensee MDPI, Basel, Switzerland. This article is an open access article distributed under the terms and conditions of the Creative Commons Attribution (CC BY) license (http:/ / creativecommons.org/licenses/by/4.0/). 